\title{
Controlling fluorescence lifetime of rare-earth element in amorphous inorganic solids via very small compositional adjustments
}

\author{
Yong Gyu Choi ${ }^{\mathrm{a})}$ \\ Department of Materials Science and Engineering, Hankuk Aviation University, Goyang, \\ Gyeonggi 412-791, Republic of Korea \\ Richard J. Curry \\ Advanced Technology Institute, School of Electronics and Physical Sciences, University of Surrey, \\ Guildford GU2 7XH, United Kingdom \\ Bong Je Park \\ Basic Research Laboratory, Electronics and Telecommunications Research Institute, Daejeon 305-600, \\ Republic of Korea \\ Kyong Hon Kim \\ Department of Physics, Inha University, Incheon 402-751, Republic of Korea \\ Jong Heo \\ Department of Materials Science and Engineering, Pohang University of Science and Technology, Pohang, \\ Gyeongbuk 790-784, Republic of Korea \\ Dan W. Hewak \\ Optoelectronics Research Centre, University of Southampton, Southampton SO17 1BJ, United Kingdom
}

(Received 25 February 2005; accepted 14 June 2005; published online 1 August 2005)

Fluorescence lifetime of hypersensitive $4 f-4 f$ transitions of rare-earth elements embedded in amorphous inorganic solids can be dramatically modified by compositional adjustment of the hosts tantamount to not more than $1 \mathrm{~mol} \%$ without any elaborated thermal treatments. It is possible to modify a spontaneous emission rate of $\mathrm{Dy}^{3+}:\left({ }^{6} \mathrm{~F}_{11 / 2},{ }^{6} \mathrm{H}_{9 / 2}\right) \rightarrow{ }^{6} \mathrm{H}_{15 / 2}$ transition in chalcogenide GeAs-S glasses through selective addition of low levels of $\mathrm{Ga}$ and $\mathrm{CsBr}$. Along with the change of the spontaneous emission rate, multiphonon relaxation rate involved in the $\left({ }^{6} \mathrm{~F}_{11 / 2},{ }^{6} \mathrm{H}_{9 / 2}\right)$ state also significantly varies upon the minute compositional adjustment. The combination of these effects results in the measured lifetime of the fluorescing $\left({ }^{6} \mathrm{~F}_{11 / 2},{ }^{6} \mathrm{H}_{9 / 2}\right)$ level being greatly enhanced. Such behaviors are attributed to the hypersensitive nature associated with the ${ }^{6} \mathrm{H}_{15 / 2} \leftrightarrow{ }^{6} \mathrm{~F}_{11 / 2}$ transition and preferential coordination of bromine in the nearest-neighboring shell of the Dy ${ }^{3+}$ ions, which is formed spontaneously during the vitrification process of the host materials. These experimental observations show the most extreme dependence of the fluorescence lifetime on small compositional changes reported compared to any other noncrystalline solid-state dielectric. As such coutilization of many hypersensitive transitions of rare-earth elements and those host materials used in this study may present a unique opportunity to control absorption and emission properties, especially fluorescence lifetimes, through a minute compositional adjustment. () 2005 American Institute of Physics. [DOI: 10.1063/1.1996854]

\section{INTRODUCTION}

The ability to control the spontaneous emission rate (SER) of a point dipole impurity embedded in a wide variety of solid-state dielectrics is a key issue to be resolved. If this can be achieved it opens up new possibilities for the application of doped dielectrics in the quantum optics field in addition to their existing applications. ${ }^{1-3}$ A rare-earth element (REE) can be regarded as a good electric dipole photonemitting source. ${ }^{4-6}$ The intra- $4 f$-configurational transitions of REE are particularly interesting as emission at various wavelengths ranging from deep ultraviolet to midinfrared can, in principle, be obtained. Tailoring SER of a REE by positioning it inside appropriate periodic arrays of a dielectric sur-

\footnotetext{
a) Author to whom correspondence should be addressed; electronic mail: ygchoi@hau.ac.kr
}

rounding is possible, although fabrication of such dielectric microstructures is usually not trivial. ${ }^{7}$ In this respect, it is important to search for solid-state dielectrics doped with REE inside which the SER is very sensitive to changes of the nearest-neighboring atoms surrounding it. Control of the SER would then be possible in such doped dielectrics by way of adjusting external conditions such as temperature, pressure, and electric field.

Due to the shielding of the REE $4 f$ electrons by the outer-lying $6 s$ and $5 d$ configurations, oscillator strengths of the $4 f-4 f$ transitions are relatively insensitive to the local coordination structure compared to the case of transitionmetal elements. Again, as the perturbation of the crystal field to the $4 f$ electronic configuration is weak, the oscillator strengths of the forced electric dipole transitions are not large; typically in the order of $10^{-6}$ in usual noncrystalline 


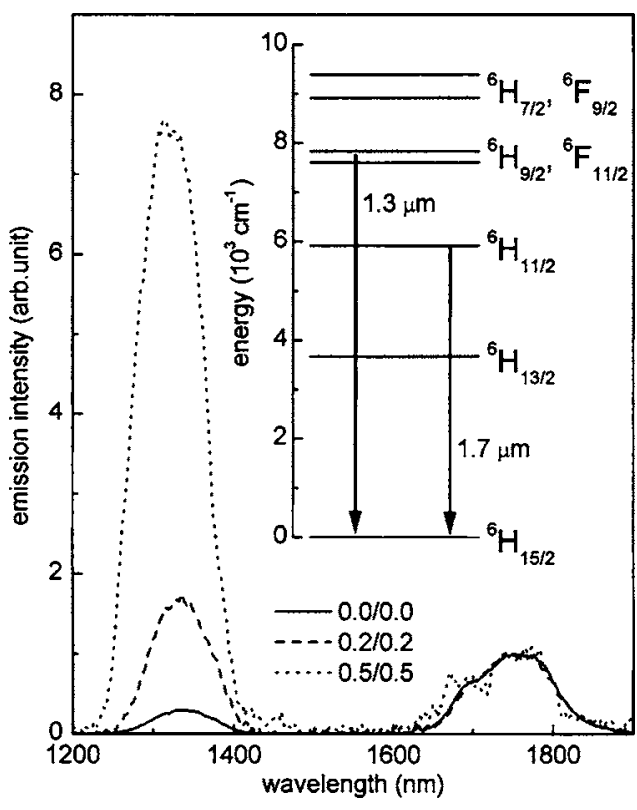

FIG. 1. Fluorescence emission spectra of some of the modified GAS glasses in the As-rich region. Note that the pump wavelength was at $800 \mathrm{~nm}$ and the intensities were normalized with respect to that of the ${ }^{6} \mathrm{H}_{11 / 2} \rightarrow{ }^{6} \mathrm{H}_{15 / 2}$ transition. The inset denotes the schematic energy-level structure of $\mathrm{Dy}^{3+}$ in the GAS glasses.

solid hosts. In an amorphous solid host, the SER of a $4 f-4 f$ transition can be changed through modification of either the crystal field at REE sites or the dielectric constant of the host medium. Typically such modification arises as a consequence of compositional change of the host. As a result the extent of the structural modification, and especially the dielectric constant, is usually assumed to be proportional to the size of the constituent change. This reasoning then requires that a significant compositional adjustment is necessary to observe any sizable change in the absorption and emission properties of REE. Some $4 f-4 f$ transitions, however, exhibit exceptional sensitivity towards local structural changes around the REE and are labeled hypersensitive. ${ }^{8,9}$ Among the $4 f$ configurations of $\mathrm{Dy}^{3+}$, the ${ }^{6} \mathrm{H}_{15 / 2} \leftrightarrow{ }^{6} \mathrm{~F}_{11 / 2}$ transition satisfies the selection rules for the hypersensitive transition that are $|\Delta J|$ $\leqslant 2,|\Delta L| \leqslant 2$, and $\Delta S=0$ for two ${ }^{2 S+1} L_{J}$ manifolds, while the ${ }^{6} \mathrm{H}_{15 / 2} \leftrightarrow{ }^{6} \mathrm{H}_{9 / 2}$ transition does not. This is significant as the ${ }^{6} \mathrm{H}_{9 / 2}$ and ${ }^{6} \mathrm{~F}_{11 / 2}$ levels are of similar energy and usually considered together and thus denoted as the $\left({ }^{6} \mathrm{H}_{9 / 2},{ }^{6} \mathrm{~F}_{11 / 2}\right)$ level. The hypersensitive nature of the Dy ${ }^{3+}:{ }^{6} \mathrm{H}_{15 / 2} \leftrightarrow{ }^{6} \mathrm{~F}_{11 / 2}$ transition was first observed experimentally from $\mathrm{Dy}^{3+}$ ions immersed in some liquids. ${ }^{10}$ Since then reports of the hypersensitive nature of this transition have been infrequent. ${ }^{11}$ In the case of noncrystalline inorganic solid hosts reported changes of the spectroscopic properties due to local structural modification around the REE have been modest, even for the hypersensitive transitions. ${ }^{12}$

Multiphonon relaxation rate (MPR) should also be considered in addition to the hypersensitive nature of the ${ }^{6} \mathrm{H}_{15 / 2} \leftrightarrow{ }^{6} \mathrm{~F}_{11 / 2}$ transition. The MPR is dominant in determining the measured lifetime of the $\left({ }^{6} \mathrm{H}_{9 / 2},{ }^{6} \mathrm{~F}_{11 / 2}\right)$ level in an ordinary sulfur-based chalcogenide glass due to the presence of the small energy gap between the $\left({ }^{6} \mathrm{H}_{9 / 2},{ }^{6} \mathrm{~F}_{11 / 2}\right)$ and the next lower-lying ${ }^{6} \mathrm{H}_{11 / 2}$ levels (see inset of Fig. 1). Such strong MPR has been the major problem associated with practicality of the $1.3-\mu \mathrm{m}$ emission of $\mathrm{Dy}^{3+}$, despite possessing some superior characteristics to the ${ }^{1} \mathrm{G}_{4} \rightarrow{ }^{3} \mathrm{H}_{5}$ transition of $\mathrm{Pr}^{3+}$ which is utilized in commercial fluoride optical fiber devices. ${ }^{13}$ The measured lifetime of the $1.3-\mu \mathrm{m}$ emission in some representative sulfide glasses is only $\sim 35 \mu$ s indicating the quantum efficiency is quite low. ${ }^{14,15}$ To reduce the MPR low-phonon-energy hosts are often considered. However, in general it is observed that the thermal and mechanical stabilities of a host medium get worse as the phonon energy of the host is lowered. It has been shown that addition of at least $10 \mathrm{~mol} \%$ of $\mathrm{CsBr}$ can be successful in increasing the lifetime of the $\mathrm{Dy}^{3+}: 1.3-\mu \mathrm{m}$ emission in a $\mathrm{Ge}-\mathrm{Ga}-\mathrm{S}$ based glass. ${ }^{16}$ Unfortunately, such significant modification on the host composition tends to deteriorate the thermal and mechanical properties. As such we postulate that such a constituent modification (a few mol \%) alters the parent glass structure itself as well as the local structure of those surrounding the REE. In addition to such structural changes we should also take into consideration the resulting localized change of the dielectric constant. Such changes affect both the optical density of state and the local-field correction factor for the absorption and emission phenomena of an electric dipole impurity in a sensitive manner. ${ }^{17}$ Based on these considerations, in this work, we aimed at tailoring only the local structure in the vicinity of REE through reducing the amount of compositional modification down to much lower doping levels. More specifically, the modification of the local structural environment of dysprosium embedded in the wellknown Ge-As-S (GAS) glasses has been made by incorporating $\mathrm{Ga}$ and $\mathrm{CsBr}$, which then exemplifies the significant role of the chemical environment of REE in controlling its radiative and nonradiative processes.

\section{EXPERIMENTAL PROCEDURE}

We fabricated Dy-doped GAS glasses modified with or without $\mathrm{Ga}, \mathrm{Cs}$, and $\mathrm{Br}$. All the starting materials were in their element forms apart from $\mathrm{Cs}$ and $\mathrm{Br}$ which were in their compound form, $\mathrm{CsBr}$. The purity of the starting materials were all $>99.999 \%$ aside from $\mathrm{CsBr}$ of $>99.9 \%$ purity. A rocking furnace was used for the 12-h-long melting of batches inside the sealed silica ampoules, which were subsequently quenched in air following by annealing at a suitable temperature (e.g., $\sim 490 \mathrm{~K}$ for the As-rich region samples). Our sample preparation procedure was the same as the conventional sulfide glass fabrication. ${ }^{18}$ After annealing, the samples did not undergo any further thermal treatment.

The glass transition temperature and the onset temperature of crystallization of the samples are all identical to each other within the measurement uncertainty of $\pm 2 \mathrm{~K}$. Transmission electron microscopy investigations suggest the absence of any crystalline phases inside the modified materials. Based on these results, we assume that the compositional modification carried out in this study does not bring about any apparent crystallization or reduce the thermal stability of the glasses.

$\mathrm{CsBr}$ is hygroscopic and therefore glasses containing it may be vulnerable to $\mathrm{OH}^{-}$contamination. Our modified 


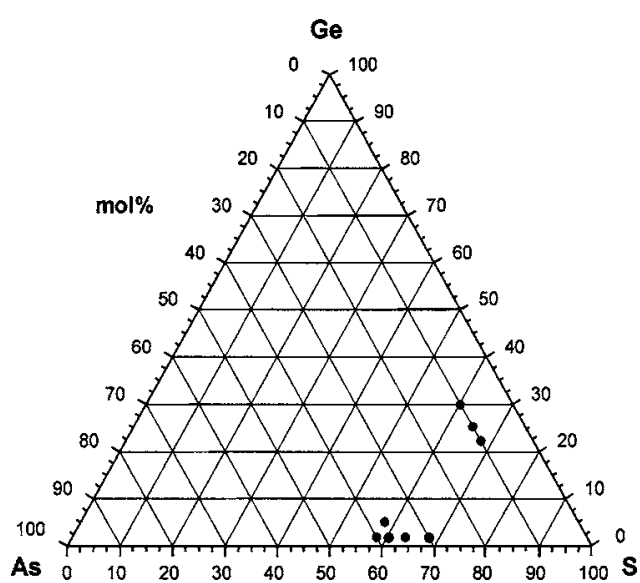

FIG. 2. The parent GAS host compositions used in this study. Ga and $\mathrm{CsBr}$ were introduced with the expense of As, while Dy replaces Ge.

GAS glasses, however, do not show any additional absorption at the infrared wavelengths corresponding to the fundamental stretching vibrations of $\mathrm{OH}^{-}$ions, which confirms that the modified glasses are free from $\mathrm{OH}^{-}$mostly due to the low concentration of $\mathrm{CsBr}$ inside.

The GAS hosts used in this study consisted of three Gerich and five As-rich glasses (Fig. 2) all doped with 0.02mol \% Dy. These compositions were chosen to make a number of $\mathrm{S}$ atoms sufficient, stoichiometric, or deficient against the $\mathrm{GeS}_{2}-\mathrm{As}_{2} \mathrm{~S}_{3}$ tie line composition. The GAS hosts modified by the inclusion of small amounts of $\mathrm{Ga}$ and $\mathrm{CsBr}$ are designated hereafter as, for example, 0.5/1.0 with each number indicating molar percentage of included $\mathrm{Ga}$ and $\mathrm{CsBr}$, respectively.

\section{RESULTS AND DISSCUSSION}

\section{A. Measured lifetimes}

Figure 3 shows the measured lifetimes of the $1.3-\mu \mathrm{m}$ emission from the $\left({ }^{6} \mathrm{H}_{9 / 2},{ }^{6} \mathrm{~F}_{11 / 2}\right) \rightarrow{ }^{6} \mathrm{H}_{15 / 2}$ transition as a function of increasing the $\mathrm{Ga}$ and $\mathrm{CsBr}$ doping concentration in equal amounts for the As-rich GAS glass (circles). Also shown are the same measured lifetimes for Ge-rich but S-sufficient, S-exact, and S-deficient glasses (square, inverted triangle, and triangle) doped with $0.5-\mathrm{mol} \% \mathrm{Ga}$ and $\mathrm{CsBr}$. In the As-rich glass the fluorescence lifetime is significantly increased upon the introduction of $\mathrm{Ga}+\mathrm{CsBr}$. The lifetime of the $0.5 / 0.5$ sample in the As-rich host is $\sim 340 \mu \mathrm{s}$, which is more than ten times the lifetime of the unmodified $0.0 / 0.0$ sample, and it further increases as long as the $\mathrm{Ga}$ $\leqslant \mathrm{CsBr}$ condition is satisfied. The condition that $\mathrm{Ga} \leqslant \mathrm{CsBr}$ in order for the lifetime to be increased is common for all the host glass compositions studied. However, the size of the lifetime enhancement is also clearly dependent on the host composition in addition to the respective concentration of $\mathrm{Ga}$ and $\mathrm{CsBr}$ with their relative ratios. The measured lifetimes of the $0.5 / 0.5$ As-rich samples are all longer than $300 \mu$ s regardless of the detailed host compositional changes. The Gerich samples, however, exhibit a different behavior, as shown in Figs. 3 and 4. With equal doping concentrations of $\mathrm{Ga}$ and $\mathrm{CsBr}$ (Fig. 4) the abrupt lifetime enhancement is observed only in a host where excess sulfur atoms are present; in this

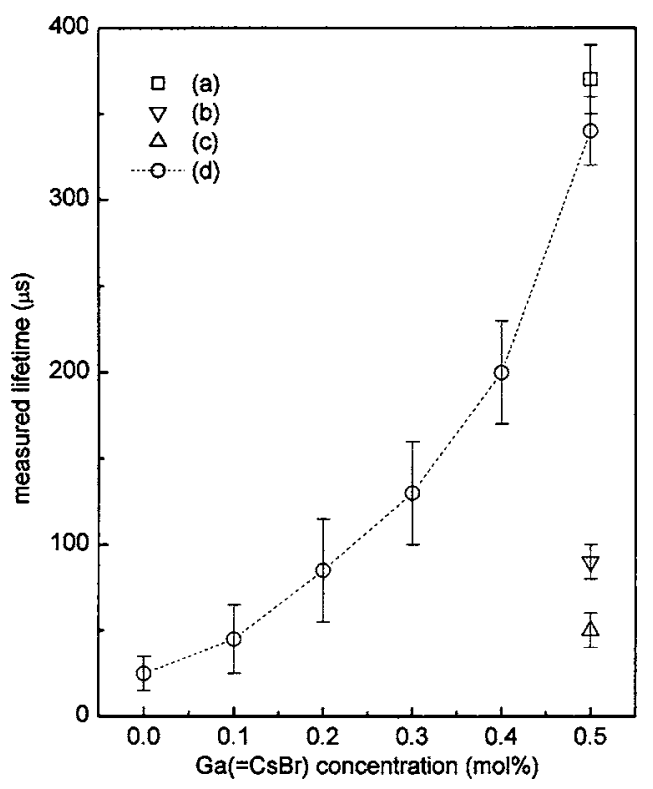

FIG. 3. Measured lifetimes of the modified GAS glasses of (a) the Ge-rich and S-sufficient, (b) the Ge-rich and S-exact, (c) the Ge-rich and S-deficient, and (d) a series of the As-rich samples. Note that the lifetimes here indicate the $e^{-1}$-folding times of the emission intensities. The error bars include the sample-to-sample variations in the measured lifetimes as well as the uncertainties involved in the measurements.

case the lifetime is measured to be $\sim 370 \mu \mathrm{s}$. Here, it should be noted that though in this case the abrupt lifetime enhancement does not appear both in the S-exact and S-deficient series, it does appear in these hosts when the doping condition $\mathrm{Ga}<\mathrm{CsBr}$ is satisfied. For example, the lifetime of $0.5 / 1.0 \mathrm{Ge}$-rich and S-exact composition is $\sim 760 \mu \mathrm{s}$.

\section{B. Oscillator strengths}

More detailed analyses have been carried out using Asrich host samples. The oscillator strengths of the 0.02-mol \%

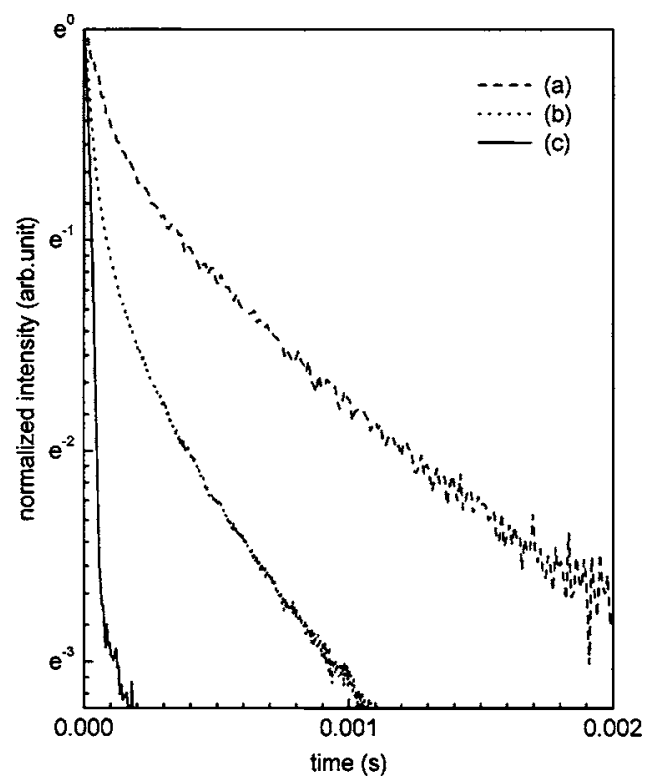

FIG. 4. Fluorescence decay profiles of the GAS glasses modified with 0.5mol \% Ga and 0.5-mol \% $\mathrm{CsBr}$ of which parent compositions are (a) the Ge-rich and S-sufficient, (b) the Ge-rich and S-exact, and (c) the Ge-rich and S-deficient samples, respectively. 


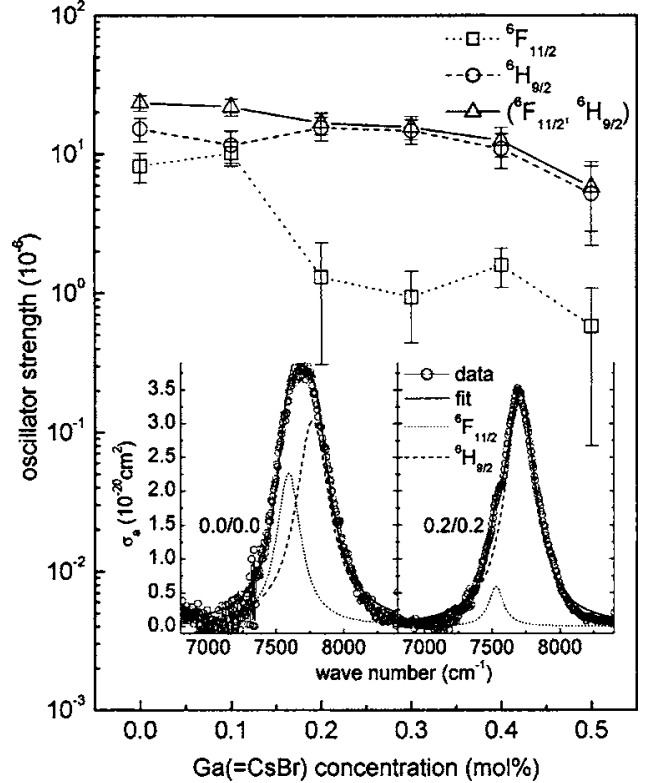

FIG. 5. Changes of oscillator strengths of each transition as a function of $\mathrm{Ga}$ $(=\mathrm{CsBr})$ concentration in a series of the As-rich GAS samples doped with 0.02-mol \% Dy. The oscillator strengths of ${ }^{6} \mathrm{H}_{15 / 2} \rightarrow{ }^{6} \mathrm{H}_{9 / 2}$ and ${ }^{6} \mathrm{H}_{15 / 2}$ $\rightarrow{ }^{6} \mathrm{~F}_{11 / 2}$ transitions were computed using the Lorentzian fit values, while that of ${ }^{6} \mathrm{H}_{15 / 2} \rightarrow\left({ }^{6} \mathrm{H}_{9 / 2},{ }^{6} \mathrm{~F}_{11 / 2}\right)$ transition was calculated directly from the measured absorption spectra. The inset shows two representative examples of the least-squares fits to the absorption cross-section spectra.

Dy-doped samples shown in Fig. 5 were calculated using their absorption cross-section spectra. ${ }^{19}$ The two closely spaced levels, ${ }^{6} \mathrm{H}_{9 / 2}$ and ${ }^{6} \mathrm{~F}_{11 / 2}$, are least-squares fitted with respective Lorentzian line-shape functions based on the assumption that the electronic states are solely and independently represented by the corresponding term symbols and inhomogeneous broadening is moderate throughout the samples. The least-squares fits with a Lorentzian profile are found to be quite reproducible and a good approximation to the bandwidth of the crystal-field splitting which is comparable with the energy discrepancy between the centers of gravity of the two levels. ${ }^{20,21}$ The overall oscillator strength of the ${ }^{6} \mathrm{H}_{15 / 2} \rightarrow\left({ }^{6} \mathrm{H}_{9 / 2},{ }^{6} \mathrm{~F}_{11 / 2}\right)$ transition decreases with increasing $\mathrm{Ga}-\mathrm{CsBr}$ concentration. While the decrease in magnitude of oscillator strength of the ${ }^{6} \mathrm{H}_{15 / 2} \rightarrow{ }^{6} \mathrm{H}_{9 / 2}$ transition is moderate, that of the hypersensitive ${ }^{6} \mathrm{H}_{15 / 2} \rightarrow{ }^{6} \mathrm{~F}_{11 / 2}$ transition abruptly decreases for $\mathrm{Ga}(=\mathrm{CsBr})$ concentrations of above $0.1 \mathrm{~mol} \%$. At these concentrations the "normal" ${ }^{6} \mathrm{H}_{15 / 2}$ $\rightarrow{ }^{6} \mathrm{H}_{9 / 2}$ transition is dominant in the overall oscillator strength. In the $0.5 / 0.5$ sample in the As-rich series, for example, the oscillator strength of the ${ }^{6} \mathrm{H}_{15 / 2} \rightarrow{ }^{6} \mathrm{~F}_{11 / 2}$ transition is reduced to $\sim 6 \%$ of that measured in the unmodified parent glass. It is interesting to note that the sudden decrease of oscillator strength of the ${ }^{6} \mathrm{H}_{15 / 2} \rightarrow{ }^{6} \mathrm{~F}_{11 / 2}$ transition occurs when the concentration of $\mathrm{CsBr}$ exceeds ten times that of the Dy atoms. Another series of samples with the same host composition but doped with $0.01-\mathrm{mol} \%$ dysprosium have also exhibited this tendency. This would indicate that the coordination number in the nearest-neighboring shell of $\mathrm{Dy}^{3+}$ is between 5 and 10, which needs to be further confirmed.

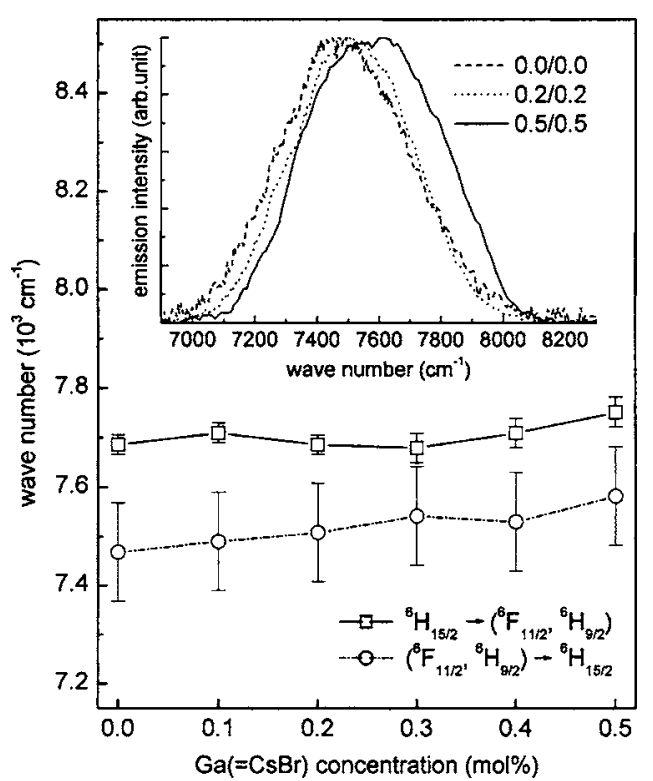

FIG. 6. The peak positions of the absorption ${ }^{6} \mathrm{H}_{15 / 2} \rightarrow\left({ }^{6} \mathrm{H}_{9 / 2},{ }^{6} \mathrm{~F}_{11 / 2}\right)$ and emission $\left({ }^{6} \mathrm{H}_{9 / 2},{ }^{6} \mathrm{~F}_{11 / 2}\right) \rightarrow{ }^{6} \mathrm{H}_{15 / 2}$ transitions. The inset denotes some representative normalized emission spectra of the As-rich GAS samples.

\section{Emission spectra}

As the oscillator strength of the ${ }^{6} \mathrm{H}_{15 / 2} \leftrightarrow{ }^{6} \mathrm{~F}_{11 / 2}$ transition is found to be very sensitive to the concentration of Ga and $\mathrm{CsBr}$ when probed by the changes of the absorption crosssection spectra, it is reasonable to expect the emission spectra to behave correspondingly. As shown in Fig. 6, the peak wavelength of the emission spectra blueshifts as $\mathrm{Ga}$ $(=\mathrm{CrBr})$ concentration increases. With this increasing concentration, the oscillator strength of the lower-energy ${ }^{6} \mathrm{H}_{15 / 2} \leftrightarrow{ }^{6} \mathrm{~F}_{11 / 2}$ transition reduces compared to that of the higher-energy ${ }^{6} \mathrm{H}_{15 / 2} \leftrightarrow{ }^{6} \mathrm{H}_{9 / 2}$ transition. As a result emission from the ${ }^{6} \mathrm{H}_{9 / 2} \rightarrow{ }^{6} \mathrm{H}_{15 / 2}$ transition becomes to dominate in the overall $\left({ }^{6} \mathrm{H}_{9 / 2},{ }^{6} \mathrm{~F}_{11 / 2}\right) \rightarrow{ }^{6} \mathrm{H}_{15 / 2}$ transition. As such the peak position of the ${ }^{6} \mathrm{H}_{15 / 2} \leftrightarrow\left({ }^{6} \mathrm{H}_{9 / 2},{ }^{6} \mathrm{~F}_{11 / 2}\right)$ absorption and emission is seen to blueshift with an increasing amount of the constituent modification. ${ }^{22}$ Such behavior is also observed from the Ge-rich series of samples.

\section{Structural changes}

The significant changes observed in the absorption and emission properties of the ${ }^{6} \mathrm{H}_{15 / 2} \leftrightarrow\left({ }^{6} \mathrm{H}_{9 / 2},{ }^{6} \mathrm{~F}_{11 / 2}\right)$ transition in our modified glasses can have a number of origins. From the viewpoint of the local structural rearrangement around $\mathrm{Dy}^{3+}$ sites, changes in coordinating atoms, interatomic distances, and their inversion symmetries have to be considered, while the microscopic dielectric constant and volume of the structurally modified region can also have an influence. Concerning the component atoms of our samples, the existence of $\mathrm{Ga}$ and $\mathrm{CsBr}$ along with their relative concentrations is a critical prerequisite to invoke the effects observed. In this regard, we have investigated structural correlation between $\mathrm{Ga}$ and $\mathrm{Br}$. The presence of $\mathrm{Cs}$ is found to play a less important role as we have obtained similar results when Cs is replaced with $\mathrm{K}$ or $\mathrm{Rb}$. Based on Raman and extended $\mathrm{x}$-rayabsorption fine structure (EXAFS) spectroscopic analyses of 
the samples modified with equal amount of $\mathrm{Ga}$ and $\mathrm{CsBr}$ in the As-rich region, gallium in the modified glasses is believed to form $\left[\mathrm{GaS}_{3 / 2} \mathrm{Br}\right]^{-}$complexes. A more detailed explanation on how $\mathrm{Br}$ ions are associated with $\mathrm{Ga}$ sulfide can be found in a previous report. ${ }^{21}$ Formation of such complexes needs to be charge compensated, and in this case $\mathrm{Dy}^{3+}$ would act as a charge compensator together with $\mathrm{Cs}^{+}$. In this way incorporation of $\mathrm{Dy}^{3+}$ close to the $\mathrm{Ga}-\mathrm{Br}$ related complexes is achieved without high-temperature heat treatment being applied. Thus we presume that preferential highly symmetric coordination in nearest-neighboring shell of dysprosium with bromine in the $\mathrm{Ga}-\mathrm{Br}$ related complexes is mainly responsible for these reported behaviors. According to the previous experimental results reported for hypersensitive transitions, a decreased oscillator strength can result from enhancing the localized inversion symmetry. ${ }^{9}$ Therefore we suggest that the local coordination structure around Dy ${ }^{3+}$ should possess enhanced site symmetry following the introduction of $\mathrm{Ga}$ and $\mathrm{CsBr}$.

As previously mentioned, the lifetime enhancement effect as a consequence of locating $\mathrm{Dy}^{3+}$ close to the $\mathrm{Ga}-\mathrm{Br}$ related complexes depends not only on the concentration of $\mathrm{Ga}$ and $\mathrm{CsBr}$ but also on the host composition, though a clear understanding of the underlying mechanisms remains to be resolved. However, differences in the chemical bond energies of the heteropolar $\mathrm{Ge}-\mathrm{S}$ and As-S bonds as well as homopolar $\mathrm{Ge}-\mathrm{Ge}$, As-As, and S-S bonds along with their relative ratios would result in such a dependence on the host composition.

\section{E. Changes of spontaneous emission rate}

A small change in the oscillator strength of the hypersensitive ${ }^{6} \mathrm{H}_{15 / 2} \leftrightarrow{ }^{6} \mathrm{~F}_{11 / 2}$ transition gives rise to a much greater change of SER for the ${ }^{6} \mathrm{~F}_{11 / 2} \rightarrow{ }^{6} \mathrm{H}_{15 / 2}$ transition compared to that for the normal ${ }^{6} \mathrm{H}_{9 / 2} \longrightarrow{ }^{6} \mathrm{H}_{15 / 2}$ transition. In the framework of Judd-Ofelt theory, ${ }^{23,24}$ the line strength for electric dipole transitions is proportional to the reduced matrix elements $\left\langle A\left\|U^{(\lambda)}\right\| B\right\rangle^{2}$ between the two electronic states involved $\langle A|$ and $|B\rangle$, where $U^{(\lambda)}$ is the tensor operator of rank $\lambda=2$, 4, or 6 . The three values of the reduced matrix elements are 0.9394, 0.8299, and 0.2061, respectively, for the ${ }^{6} \mathrm{~F}_{11 / 2} \rightarrow{ }^{6} \mathrm{H}_{15 / 2}$ transition, and $0.0000,0.0166$, and 0.2017 for the ${ }^{6} \mathrm{H}_{9 / 2} \rightarrow{ }^{6} \mathrm{H}_{15 / 2}$ transition. ${ }^{25}$ The values for transitions from the ${ }^{6} \mathrm{~F}_{11 / 2}$ state to other remaining underlying states (Fig. 1) are also larger than the corresponding cases for the ${ }^{6} \mathrm{H}_{9 / 2}$ state. As such SER of the ${ }^{6} \mathrm{~F}_{11 / 2}$ level is much more sensitive to the changes of the oscillator strengths than that of the ${ }^{6} \mathrm{H}_{9 / 2}$ transition. Thus, the decreased oscillator strengths of the ${ }^{6} \mathrm{H}_{15 / 2} \leftrightarrow{ }^{6} \mathrm{~F}_{11 / 2}$ transition, which arises as a consequence of the local structural changes at the $\mathrm{Dy}^{3+}$ sites, results in a significant decrease of SER of the ${ }^{6} \mathrm{~F}_{11 / 2}$ state in the modified GAS glasses. On the other hand the refractive index measured at $1.3 \mu \mathrm{m}$ is found to decrease with increasing concentrations of $\mathrm{Ga}$ and $\mathrm{CsBr}$, though the corresponding changes are all less than $\sim 0.02$. Therefore the changes in the dielectric constant would not fully account for the observed SER changes, though the microscopic dielectric constant around the $\mathrm{Dy}^{3+}$ is not clearly known at this time.
It is worthwhile to note that the uncertainty of JuddOfelt analysis usually becomes larger when hypersensitive transitions are involved. In addition application of the Lorentzian-type local-field correction factor, which has been most widely used, may fail to account for the actual local field experienced by the doped impurity dipoles inside solid dielectrics. ${ }^{26}$ We therefore ruled out the results of Judd-Ofelt analyses on our samples. On the other hand, the FuchtbauerLadenburg equation was considered to be inadequate to calculate the intrinsic lifetime of the $\left({ }^{6} \mathrm{H}_{9 / 2}{ }^{6} \mathrm{~F}_{11 / 2}\right)$ level in that the ${ }^{6} \mathrm{H}_{15 / 2}$ and $\left({ }^{6} \mathrm{H}_{9 / 2},{ }^{6} \mathrm{~F}_{11 / 2}\right)$ levels are not just a two-level system but there are two other energy levels between them, as shown in Fig. 1.

\section{F. Changes of multiphonon relaxation rate}

Together with the decreased SER, the reduced MPR is also responsible for the far enhanced lifetimes of the 1.3$\mu \mathrm{m}$ emission in our modified glasses. Population of the ${ }^{6} \mathrm{H}_{11 / 2}$ level is fed from the above-lying $\left({ }^{6} \mathrm{H}_{9 / 2},{ }^{6} \mathrm{~F}_{11 / 2}\right)$ level mainly through multiphonon relaxation processes. The intensity of the $1.3-\mu \mathrm{m}$ emission increases compared to the 1.7$\mu \mathrm{m}$ emission from the ${ }^{6} \mathrm{H}_{11 / 2} \rightarrow{ }^{6} \mathrm{H}_{15 / 2}$ transition with increasing $\mathrm{Ga}(=\mathrm{CsBr})$ concentration as shown in Fig. 1. This evidence clearly indicates that the decreased MPR in the modified samples must be taken into consideration. The Raman spectra reveal that a molecular vibration associated with the $\mathrm{Ga}-\mathrm{Br}$ chemical bond appears at $\sim 260 \mathrm{~cm}^{-1}$ in the modified samples, ${ }^{21,27}$ which is smaller than the representative phonon energy of these sulfide glasses, $\sim 350 \mathrm{~cm}^{-1} \cdot{ }^{18}$ In this regard we postulate that as we surround the $\mathrm{Dy}^{3+}$ with $\mathrm{Br}$ we are reducing the local phonon energy around the ion from that of the host glass $\left(\sim 350 \mathrm{~cm}^{-1}\right)$ to $\sim 260 \mathrm{~cm}^{-1}$, and then the $\sim 260-\mathrm{cm}^{-1}$ phonons participate in the MPR process of the $\left({ }^{6} \mathrm{H}_{9 / 2},{ }^{6} \mathrm{~F}_{11 / 2}\right)$ level in the modified glasses.

\section{G. Changes of electronic band-gap energy}

Besides the reported abrupt changes of the spectroscopic properties of $\mathrm{Dy}^{3+}$, the electronic absorption edge of the host glass itself is significantly changed as $\mathrm{Ga}$ and $\mathrm{CsBr}$ are introduced (Fig. 7). The band-gap energy of the Ge-rich glasses blueshifts upon the introduction of such small amounts of $\mathrm{Ga}$ and $\mathrm{CsBr}$, while that of the As-rich glasses redshifts upon the same constituent modification. Though the bulk thermal properties of the host glasses remain unchanged following the introduction of small amounts of $\mathrm{Ga}$ and $\mathrm{CsBr}$, it is perhaps more surprising that the band-gap energy of the host glass is modified to such an extent.

\section{CONCLUSION}

In summary, we observe that it is possible to modify fluorescence lifetime of the $\left({ }^{6} \mathrm{H}_{9 / 2},{ }^{6} \mathrm{~F}_{11 / 2}\right)$ states of $\mathrm{Dy}^{3+}$ in noncrystalline $\mathrm{Ge}-\mathrm{As}-\mathrm{S}$ alloys through the selective addition of low levels of $\mathrm{Ga}$ and $\mathrm{CsBr}$. We show that the change in SER + MPR is more sensitive to the local coordination structure of the Dy ${ }^{3+}$ compared to other hypersensitive transitions that have been observed so far in solid-state dielectric hosts. Preferential highly symmetric coordination in nearestneighboring shell of dysprosium with bromine in 


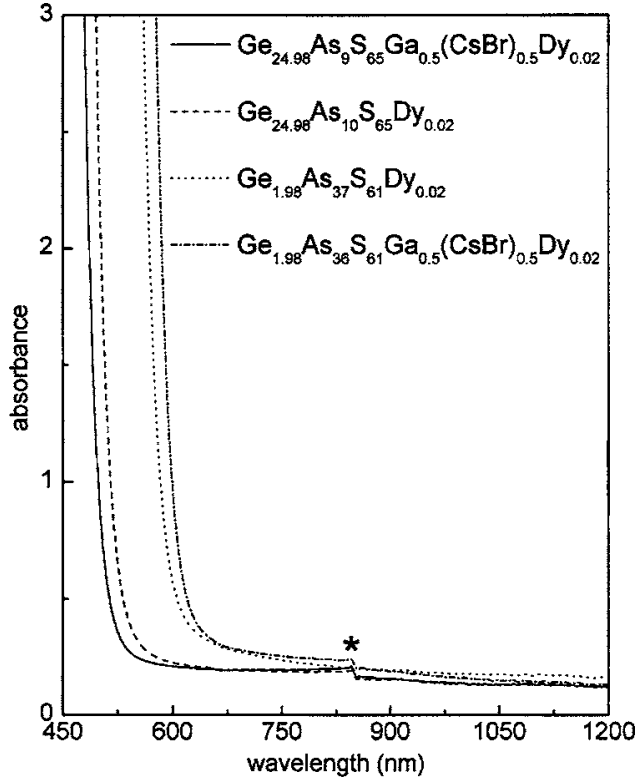

FIG. 7. Absorption spectra of some selected samples. Note that the steps at $\sim 760 \mathrm{~nm}$ marked with an asterisk resulted from an instrumental feature.

$\left[\mathrm{GaS}_{3 / 2} \mathrm{Br}\right]^{-}$complexes, which are formed spontaneously during the vitrification process of the host materials, is believed to be responsible for the behavior. As such the control of absorption and emission properties, especially fluorescence lifetimes, is possible through a minute compositional adjustment. Coutilization of many hypersensitive transitions of rare-earths elements and those host materials used in this study thus present such an opportunity.

\section{ACKNOWLEDGMENT}

This work was made possible by the financial support from the Korea/UK joint collaboration program and the Ministry of Information and Communication, Republic of Korea.
E. M. Purcell, Phys. Rev. 69, 681 (1946).

${ }^{2}$ K. H. Drexhage, J. Lumin. 1-2, 693 (1970).

${ }^{3}$ P. Lodahl, A. Floris van Driel, I. S. Nikolaev, A. Irman, K. Overgaag, D. Vanmaekelbergh, and W. L. Vos, Nature (London) 430, 654 (2004).

${ }^{4}$ F. J. P. Schuurmans, D. T. N. de Lang, G. H. Wegdam, R. Sprik, and A. Lagendijk, Phys. Rev. Lett. 80, 5077 (1998).

${ }^{5}$ P. Lavallard, M. Rosenbauer, and T. Gacoin, Phys. Rev. A 54, 5450 (1996).

${ }^{6}$ G. L. J. A. Rikken and Y. A. R. R. Kessener, Phys. Rev. Lett. 74, 880 (1995).

${ }^{7}$ A. M. Vredenberg, N. E. J. Hunt, E. F. Schubert, D. C. Jacobson, J. M. Poate, and G. J. Zydzik, Phys. Rev. Lett. 71, 517 (1993).

${ }^{8}$ R. D. Peacock, Struct. Bonding (Berlin) 22, 83 (1975).

${ }^{9} \mathrm{C}$. Görller-Walrand and K. Binnemans, in Handbook on the Physics and Chemistry of Rare Earths, edited by K. A. Gschneidner, Jr. and L. Eyring (Elsevier, Amsterdam, 1998), Vol. 25, p. 220.

${ }^{10}$ W. T. Carnall, J. Phys. Chem. 67, 1206 (1963).

${ }^{11}$ M. B. Saisudha and J. Ramakrishna, Phys. Rev. B 53, 6186 (1996).

${ }^{12}$ V. K. Tikhomirov, M. Naftaly, and A. Jha, J. Appl. Phys. 86, 351 (1999).

${ }^{13}$ Y. Ohishi, T. Kanamori, T. Nishi, and S. Takahashi, IEEE Photonics Technol. Lett. 3, 715 (1991).

${ }^{14}$ B. N. Samson, J. A. Medeiros Neto, R. I. Laming, D. W. Hewak, and D. N. Payne, Electron. Lett. 33, 414 (1994).

${ }^{15}$ J. Heo and Y. B. Shin, J. Non-Cryst. Solids 196, 162 (1996).

${ }^{16}$ Y. B. Shin, J. Heo, and H. S. Kim, Chem. Phys. Lett. 317, 637 (2000).

${ }^{17}$ W. B. Fowler and D. L. Dexter, Phys. Rev. 128, 2154 (1962).

${ }^{18}$ Y. G. Choi, K. H. Kim, S. H. Park, and J. Heo, J. Appl. Phys. 88, 3832 (2000).

${ }^{19}$ Y. G. Choi and J. Heo, J. Non-Cryst. Solids 217, 199 (1997).

${ }^{20}$ J. B. Gruber, B. Zandi, B. Justice, and E. F. Westrum, Jr, J. Chem. Phys. 110, 12125 (1999).

${ }^{21}$ Y. G. Choi, R. J. Curry, B. J. Park, K. H. Kim, J. Heo, and D. W. Hewak, Chem. Phys. Lett. 403, 29 (2005)

${ }^{22}$ D. E. McCumber, Phys. Rev. 136, 954 (1964).

${ }^{23}$ B. R. Judd, Phys. Rev. 127, 750 (1962).

${ }^{24}$ G. S. Ofelt, J. Chem. Phys. 37, 511 (1962).

${ }^{25}$ W. T. Carnall, H. Crosswhite, and H. M. Crosswhite, Energy level structure and transition probabilities of the trivalent lanthanides in $\mathrm{LaF}^{3}$, Report No. ANL-78-XX-95, Argonne National Laboratory, Chemistry Division, Illinois, 1978

${ }^{26}$ G. M. Kumar, D. N. Rao, and G. S. Agarwal, Phys. Rev. Lett. 91, 203903 (2003).

${ }^{27}$ Yu. S. Tver'yanovich, M. Vlček, and A. Tverjanovich, J. Non-Cryst. Solids 333, 85 (2004) 